\title{
Theaterpädagogische Elemente in \\ Fremdsprachenunterricht integrieren - am Beispiel eines Kiswahili-Kurskonzepts
}

\author{
Susanne Horstmann
}

\begin{abstract}
Zusammenfassung
Der Artikel beschäftigt sich mit der Frage, wie man Fremdsprachenunterricht so gestalten kann, dass die ursprünglich bei allen Menschen vorhandene Neugier und Lust am Lernen wieder geweckt wird, wie man Aspekte natürlichen ungesteuerten L2-Lernens auch im Fremdsprachenunterricht simulieren kann und wie Spielelemente auch mit spielungewohnten Gruppen so in den Unterricht integriert werden können, dass das Spiel ein wirkliches Spiel wird. Außerdem spielen die Reduktion von Sprechangst und die Förderung von Lernerautonomie insbesondere hinsichtlich bevorzugter Sprachlernwege eine Rolle. Dazu werden im vorgestellten Konzept sowohl Bewegung, Rhythmus, Melodie und Rollen- und Theaterspielelemente genutzt als auch Clownsprinzipien. Die Herangehensweise ist für das deutsche Bildungssystem unüblich und will einen Kontrapunkt zum Anspruch der Ver-Messbarkeit von allem setzen.
\end{abstract}

\section{Einleitung}

„... doch etwas ganz Zentrales scheint mir zunehmend verloren zu gehen: die Radikalität, die Parteilichkeit, die Skepsis im theaterpädagogischen Arbeiten. Die Skepsis gegenüber der Welt ist die Voraussetzung für ein kritisches Bewusstsein" (Nix 2012: 45)

Ein solches Bewusstsein sollte m.E. jeder theaterpädagogischen Arbeit zugrunde liegen: Sie sollte Lerner*innen selbstständiger machen, kritischer und stärker, und sie sollte versuchen, Gegengewichte zu strukturellen Zwängen zu setzen, wachzumachen, zu zeigen, dass Lernen und Zusammenarbeiten auch anders funktionieren können, als wir das für unser Bildungssystem zumindest heimlich noch immer als gültig annehmen. Genauer: Mit meinem hier vorgestellten Ansatz möchte ich zeigen, dass man auch in institutionellem Raum scheinbar selbstverständliche Muster von Fremdsprachenunterricht aufbrechen kann, dass Teilnehmer*innenorientierung nicht nur ein Lippenbekenntnis sein muss, dass Lerner*innen mitentscheiden können, was und wie sie lernen und dass 
Lernen aus Interesse und Neugierde und mit Spaß nicht nur lernförderlich, sondern auch möglich ist (vgl. Sambanis 2013: Kap. 2, insb. S. 25-35, Holec 1981), und zwar nicht nur für einzelne, sondern für alle Teilnehmer*innen (TN) eines Kurses. Deshalb integriert der im Folgenden vorgestellte Ansatz viele Aspekte von Freiwilligkeit und Interesse der Lerner*innen, viele Angebote seitens der Lehrer*innen und immer wieder die Ermunterung einzufordern, was gebraucht wird.

Ich will aber in diesem Artikel auch auf die Reibung im und am System aufmerksam machen, nicht weil das gegen meinen Ansatz spricht, sondern weiles uns bewusst macht, dass wir das System verändern sollten. Anders gesagt: Es geht meines Erachtens nicht nur um die Frage, inwiefern sich die Theaterpädagogik an Fremdsprachenunterricht anpassen sollte (siehe Küppers \&Walter 2012: 5), sondern auch darum, inwiefern sich der Fremdsprachenunterricht an die Theaterpädagogik anpassen sollte und deren Prinzipien, Grundsätze und Möglichkeiten nutzen kann, um freundlicher und menschengerechter $\mathrm{zu}$ werden.

Wichtig für mein Kurskonzept war es, außer einer Orientierung an den Interessen der Lerner*innen und dem Versuch der Förderung selbstständigen Lernens, eine Atmosphäre der Fehlerfreundlichkeit (im Sinne von make misteaks $^{1}$ (sic!)) und der Risikobereitschaft zu schaffen (zu dem Thema siehe auch Haftner/Kuhfuß 2014: 222). Als Spracherwerbsforscher*innen wissen wir, dass Fehler notwendiger Bestandteil von Lerner*innensprachen sind, dass sie klug und hochinteressant sein können, weil sie Hypothesen über das Funktionieren der Zielsprache zeigen, dass die Angst vor Fehlern zu Sprechhemmungen führt und dass das Aufmerksam-Machen auf Fehler üblicherweise keine positiven Emotionen auf Seiten der Lerner*innen weckt. Deshalb halte ich es für wichtig, Fehler zwar phasenweise zu korrigieren, insgesamt aber den Lerner*innen zu vermitteln, dass Fehler Teil des Lernprozesses und viel weniger wichtig als (kommunikativer) Gebrauch der Fremdsprache sind. Eine von allen Interaktanten verinnerlichte Fehlerfreundlichkeit führt zu einer erhöhten Bereitschaft, Risiken einzugehen, mutiger zu werden und sich auch dann zu äußern, wenn man nicht sicher ist, ob das, was man sagen will, richtig ist. Wichtig ist in einem Anfängersprachkurs an erster Stelle, sich zu trauen, in der Zielsprache sprachlich zu handeln und zu interagieren. Fehlerkorrektur kann damit dann als das gesehen werden, als das sie gemeint ist, als emotional unbelasteter, manchmal notwendiger Focus on Form, um langfristig die inhaltliche Kommunikation zu verbessern. Risikobereitschaft wiederum hilft beim Abbau von Sprechangst.

\section{Konzeption für einen Anfängersprachkurs mit theaterpädagogischen Elementen}

Das vorliegende Konzept wurde im Rahmen eines Kiswahili-AnfängerSprachkurses entwickelt und bereits teilweise auf das Deutsche übertragen.

\footnotetext{
${ }^{1}$ Quelle: Toilettenspruch an der Universität Bielefeld.
} 
Der Kiswahili-Kurs fand im Rahmen der frei wählbaren Angebote des Fachsprachenzentrums der Universität Bielefeld statt. Die 17 TN sprachen mehrheitlich Deutsch als Erstsprache, aber auch internationale Student*innen mit den Erstsprachen Chinesisch, Vietnamesisch und Französisch nahmen teil. Eine Teilnehmerin war kenianisch-deutsch, hatte also Familie in Kenia und zumindest schon des Öfteren Kiswahili gehört, ein anderer hatte ein Jahr in Ruanda verbracht und lernte Kiswahili in Ermangelung eines KinyarwandaAngebots. Der Kurs umfasste einen Zeitraum von 15 Wochen. Pro Woche fanden jeweils zwei 90-min-Einheiten statt, von denen die erste im Theaterprobenraum, einem großen leeren Raum mit Holzfußboden, und die zweite am Folgetag in einem normalen Unterrichtsraum durchgeführt wurde. Im Theaterprobenraum wurde der Schwerpunkt auf das Spielen gelegt; die Interaktion fand fast ausschließlich in der Zielsprache statt und es wurden diverse Aktivitäten durchgeführt. Die Hausaufgabe bestand nach diesen Sitzungen immer darin, alles aufzuschreiben, was man behalten hat und zu überlegen, was man am Folgetag gerne fragen möchte.

Die Unterrichtsraumsitzung am folgenden Tag wurde immer mit der Möglichkeit zu fragen eingeleitet und alle Fragen wurden, wenn auch unterschiedlich ausführlich, beantwortet. Dies ist m.E. sinnvoll für jeden Unterricht: Wenn wir Lernen mit der Lebenswelt von Lerner*innen verknüpfen und von ihren Interessen ausgehen wollen, müssen Lehrer*innen Fragen zu allen Themen auch wenn sie nicht im Curriculum ,dran' sind - beantworten. Es gibt nichts Demotivierenderes als ein „Das ist jetzt noch nicht dran - das brauchst du nicht zu wissen“. Wenn wir als Lehrer*innen selbstständige, autonome Lerner*innen wollen, müssen deren Interessen berücksichtigt werden. Nach Beantwortung der Fragen wurde ,üblicher' Fremdsprachunterricht durchgeführt. Feste Hausaufgaben für alle Kursmitglieder (außer der Reflexionsaufgabe zwischen Spiele- und Unterrichtsraumsitzung) gab es allerdings nur selten, stattdessen viele Anregungen, was und wie man noch üben könnte, und dazu wurden Materialien und Online-Links zur Verfügung gestellt. Auch dieses Vorgehen war begründet in der Autonomieförderung und dem Wunsch, an die Interessen der Lerner anzuknüpfen und ihnen bevorzugte Lernwege/Lernmöglichkeiten zur Verfügung zu stellen.

Alle Grammatik-Themen und die meisten inhaltlichen Themen wurden zunächst in den Spielesitzungen interaktiv realisiert und anschließend in den Unterrichtsraumsitzungen erläutert, hinterfragt und durch schriftliche und mündliche Aufgaben und Übungen gefestigt. Auch weitere Aktivitäten in den folgenden Spielesitzungen dienten der Festigung .

Ziel der Spielesitzungen war es, die Neugier der Lerner*innen zu wecken und durch Fehlerfreundlichkeit die eigene Experimentierfreudigkeit mit Sprache zu fördern. Um einen vom üblichen Unterricht abweichenden Rahmen zu setzen, ist es sinnvoll, die übliche Lehrer*in-Schüler*in-Relation so weit wie möglich aufzuweichen. Das heißt für die Lehrkraft, eher mitzuspielen als zu beobachten und vor allem, sich als erster und Vorbild ,zum Affen zu machen'. Man kann von den Lerner*innen nichts fordern, was man nicht selbst auch bereit ist zu geben. 
Das heißt aber auch, in den Spielesitzungen immer wieder daran zu erinnern, dass nicht die Korrektheit des Sprachgebrauchs im Fokus steht, sondern Spaß, Spiel und das Schaffen von Gemeinsamkeit innerhalb des Gruppenverbandes. Das Prinzip der Freiwilligkeit, insbesondere für Hausaufgaben, war verbunden mit dem Appell, Unterschiedliches auszuprobieren und so herauszufinden, welche Angebote das eigene Lernen begünstigen, welche Lernwege sich als effektiv für das Individuum erweisen - und sich von dieser Sorte Angebot mehr zu holen.

Abseits vom üblichen Unterricht wurde zum weiteren Abbau von Sprachhemmungen, zur Förderung von Neugierde, Fehlerfreundlichkeit und positiven Emotionen nach sechs Wochen (auf Deutsch) am Wochenende ein eintägiger Clowns-Workshop angeboten (siehe Punkt 5). Von diesem Zeitpunkt an wurden in die Spielesitzungen auch Clowns-Spiel-Aufgaben integriert.

\section{Zentrale Spielelemente des Kurskonzepts ${ }^{2}$}

a) Alle Spielesitzungen beginnen mit einem Aufwärmspiel ${ }^{3}$, bei dem selbstverständlich auch die Lehrkraft immer mitspielt. In den ersten 6 Wochen ist dies immer eine andere Sorte Fang-Spiel. Fang-Spiele haben den Vorteil, dass sie keinerlei Hemmschwelle haben, d.h. auch spielungewohnte und sogar spielskeptische Lerner*innen haben Spaß, und sie wärmen sich körperlich auf, um in den Spielmodus umzuschalten. Einige der Spiele beinhalten bereits kleinere Elemente von Körperkontakt, so z. B. Knuddelpacken, bei dem ein Fänger jemand fängt, der dann der neue Fänger wird. Schützen können sich die Weglaufenden, indem sie eine (und nur eine) weitere Person berühren ${ }^{4}$. Weitere Beispiele für Fangspiele sind:

- Kettenpacken: Gefangene fassen den ursprünglichen Fänger an und werden so auch zu Fängern. Die Kette wird immer länger; nur die Außenglieder der Kette dürfen fangen

- Baum-Stein-Höhle: Der Fänger wechselt nicht, sondern verwandelt durch Zuruf die Gefangenen in einen Baum, einen Stein oder eine Höhle. Die Verwandelten können befreit werden. Um den Baum muss jemand zur Befreiung herumlaufen, über den Stein springen und durch die Höhle krabbeln.

\footnotetext{
${ }^{2}$ Einen großen Pool an weiteren für den Fremd- und Zweitsprachenunterricht nutzbaren theaterpädagogischen Aktivitäten bietet Bohle 2011/2017.

${ }^{3}$ Weitere Aufwärmspiele finden sich z. B. unter http://improwiki.com/de/wiki/ improtheater/aufwaermspiele, 23.05.2017.

4Übungen mit Körperkontakt, jeweils sukzessive dem Stand der Gruppe entsprechend eingesetzt, dienen dazu, Hemmungen im Umgang miteinander zu überwinden und einen vom üblichen Fremdsprachenunterricht abweichenden Rahmen zu setzen sowie die positive Gruppendynamik zu fördern.
} 
- Knie fangen (Paare, jeder versucht, die Knie des Gegenübers zu berühren).

- Körperteilpacken (das zu berührende Körperteil wird gerufen).

b) Die Spielanweisungen werden von Anfang an nur in der Zielsprache gegeben, das Spiel aber auch interaktiv von den (mitspielenden) Lehrer*innen demonstriert, so dass letztlich immer von Anfang an alles problemlos verstanden werden kann. Auch sämtliche weitere Arbeitsanweisungen werden in den Spielesitzungen auf Kiswahili formuliert und nur dann von der Lehrkraft übersetzt, wenn niemand verstanden hat, was gemacht werden soll. Bereits nach wenigen Wochen gibt es immer einige Lerner*innen, die den Inhalt der Aufgaben verstehen und die Erläuterung für die anderen Lerner*innen geben können. Diese Arbeitsanweisungen sind eine Art Sprachbad. Sprachbäder können auch aus rhythmisch gesprochenen und gestisch begleiteten Texten bestehen, beispielsweise dem Nachsprech-Spiel Wir gehen jetzt auf Bärenjagd (Twaenda kuwinda). Hier kommt es nicht auf Genauigkeit und Korrektheit des Nachsprechens an, sondern auf das Nachsprechen und Herstellen einer Vertrautheit mit dem Klang der fremden Sprache überhaupt. Sprachbäder werden zunächst nicht weiter bearbeitet, d.h. Texte nicht übersetzt $\mathrm{o}$. ä. Wenn wöchentlich wiederholt, werden die Lerner*innen nach und nach einzelne Elemente identifizieren oder gar wiedererkennen und verstehen. Diese Elemente können dann im Kurs reflektiert, schriftlich festgehalten etc. werden. Die Bearbeitung geht aber von den Lerner*innen aus.

c) Verschiedene Spielarten von Total Physical Response (TPR ${ }^{5}$ ) werden insbesondere in den ersten Wochen in die Spielesitzungen integriert: Man beginnt, in dem man mit vier Bewegungsverben spielt (z. B.: lauf/ steh/ sitz/ spring/ klatsch). Zunächst macht die Lehrkraft mit, so dass den Lerner*innen die durchzuführende Aktion jeweils auch demonstriert wird. Wenn erste Lerner*innen die richtige Tätigkeit ausführen, zieht sich die Lehrkraft heraus und gibt die Befehle. Da irgendjemand immer richtig reagiert und die anderen ihn nachahmen können, stellt auch dies keine Schwierigkeit dar. Die Lerner*innen kommen auch bei dieser Übung außer Atem, die Übung dient also weiterhin dem Aufwärmen. Schließlich werden einzelne Lerner*innen aus der Gruppe gezogen, die dann die Befehle geben. Die nun die benutzten Verben können im Imperativ auf ein Flip-Chart geschrieben werden. Die Befehle sollten aber ohne ständigen Blick auf das Plakat gegeben werden ${ }^{6}$.

\footnotetext{
${ }^{5}$ Die Methode Total Physical Response wurde 1964 von dem Psychologen James Asher entwickelt und gilt als alternative Lehrmethode. M.E. ist sie im Anfängerunterricht in Verbindung mit anderen Techniken gut nutzbar, auf keinen Fall aber als einzige oder längerfristig als Hauptmethode im Fremdsprachenunterricht zu wählen. Die Methode sowie ihre Vor- und Nachteile werden übersichtlich von Teymoortash 2010 geschildert (ebd., S. 153-167 sowie S. 233ff).

${ }^{6} \mathrm{Zu}$ beachten bei der Zielsprache Deutsch ist, dass zunächst nur schwache Verben bzw. Verben, die zumindest im Präsens nicht den Stammvokal ändern, zu wählen sind oder die
} 
Zu einem späteren Zeitpunkt wird die Technik TPR wieder aufgenommen und dann durch zwei bis vier Verben nach Wahl der Lerner*innen (z. B. Hobbys: Was machst du gerne?) erweitert. Gestisch und mimisch demonstriert die Lehrkraft, dass sie beispielsweise Schwimmen mag und Joggen hasst und sie fragt die Lerner*innen, was sie gerne mögen. So werden weitere drei bis vier Begriffe pro Spieldurchgang zum Flip-Chart mit den Verben hinzugefügt. TPR wird immer mit allen vorhandenen Begriffen nach dem oben beschriebenen Muster gespielt. Eine weitere bereits am Anfang zu spielenden Hobby-Aktivität ist Alle, die gerne schwimmen/tanzen/laufen.... (selbstverständlich in der Zielsprache). Dabei sitzt die Gruppe im Kreis an festgelegten Plätzen (Stühle oder Gegenständen die Plätze markieren). Einer hat keinen Platz und ruft Alle, die gerne.... und fügt eines der Hobbys ein. Diejenigen, die das Genannte gerne machen, müssen aufstehen, eine Runde um den Kreis laufen und sich dann einen neuen Platz suchen. Da ein Platz fehlt, wird nun ein anderer außen stehen bleiben müssen und ist der/die nächste Rufer*in. Die Formel Alle, die gerne wird als Chunk gelernt und genutzt.

In späteren Stunden lässt sich die Technik TPR weiterhin mit dem Spiel Simon says (jeweils in die Zielsprache übersetzt) verbinden ${ }^{7}$. Dabei ist der Befehl nur auszuführen, wenn diesem ein „Simon says..." vorangestellt wird. Schließlich kann man mit dieser Technik die Negation einüben: Lehrer*in und Gruppe stehen einander gegenüber, die Gruppe im Pulk. Der/Die Lehrer*in gibt mit energischem Schritt nach vorne Befehl, der Kurs und weigert sich mit genauso energischem Schritt nach vorne („Geh!“ „Ich gehe nicht!“ oder „Geht!“ „Wir gehen nicht!“).

d) Weitere, nicht nur zu Beginn des Kurses genutzte Elemente sind Musik und Rhythmus. Die Gruppe steht im Kreis und zunächst wird ein Viererschritt eingeübt.

$$
\begin{aligned}
& 1 \text { - rechter } \mathrm{Fu} ß \text { vor } \\
& 2 \text { - linker } \mathrm{Fu} \text { am Platz auftippen } \\
& 3 \text { - rechter } \mathrm{Fu} ß \text { zurück } \\
& 4 \text { - linker Fuß am Platz auftippen }
\end{aligned}
$$

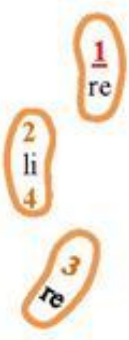

Abbildung 1: Viererschritt ${ }^{8}$

Das Einüben dauert einige Minuten, bei Bedarf können auch schon im

\footnotetext{
Infinitive genutzt werden sollten.

${ }^{7}$ Es empfiehlt sich nicht, die TPR mit Go - Stop - Clap - Jump zu verbinden, da zunächst die korrekten mentalen Repräsentationen der Wörter geschaffen werden müssen.

${ }^{8}$ Nach Mike Turnbull, Percussionist, u. a. tätig in der theaterpädagogischen Ausbildung der LAG Spiel und Theater NRW.
} 
Chor die Zahlen 1-4 mit den jeweiligen Schritten verbunden werden und/oder mit Klatschern auf Beats oder später Off-Beats. Ist die Gruppe gemeinsam im Viererrhythmus, wird die erste Zeile des Namensliedes zunächst mit Vor- und Nachsingen, dann in einer Schleife eingeübt. Das verwendete Lied basiert auf der Melodie des Chants Mother I feel you under my feet.

$\begin{array}{lll}\text { Strophe: } & \text { Jina langu mhmh } & \text { Ich heiße mhmh } \\ & \text { Jina lako nani? (WH 2x) } & \text { Und wie heißt du? } \\ & \text { Jina langu mhmh } & \text { Ich heiße mhmh } \\ & \text { Tunasalimiana } & \text { Wir grüßen einander } \\ & \text { Habari gani? } & \text { Wie geht's dir heut? } \\ \text { Refrain: } & \text { Nzuri tu (WH 1x) } & \text { Sehr gut, sehr gut } \\ & & \\ & \text { Asante sana,. } & \text { Herzlichen Dank auch, } \\ \text { 2. Stimme } & \text { Asante sana. } & \text { herzlichen Dank auch. } \\ \text { (Basso Continuo) } & \end{array}$

Abbildung 2: Liedtext „Jina langu...“, Namenslied nach der Melodie von „Mother I feel you under my feet"

Chants sind eingängig und mehrstimmig und lassen sich (umgedichtet) sehr gut für den Fremdsprachenunterricht nutzen. Sind Text und Melodie ansatzweise vertraut, singt jeder bei mhmh den eigenen Namen. Anschließend können Frage- und Antwortmuster etabliert werden und Einzelpersonen werden (immer noch in Lied, Schritt und Rhythmus) gefragt und antworten. Dabei werden zunächst viele nicht-zielsprachgerechte Formen produziert. Das ist an dieser Stelle kein Problem, es geht um ein erstes Vertraut-Werden mit dem Klang der Sprache und um ein erstes mutiges Sprechen. Rolle der/des Lehrenden ist also: Ermutigen und Loben. Die Lehrkraft kann anschließend im Kreis umhergehen und die Mini-Dialoge mit Einzelpersonen inszenieren. Hier können auf Nachfrage auch Korrekturen gegeben werden. Anschließend wird, wieder im Viererschritt, der zweite Teil des Liedes eingeführt, der Refrain. Nach einigen Hin-und-Her-Namenfragen (3-4x) wird der Refrain gesungen.

Zu einem späteren Zeitpunkt kann die Aktivität mit weiteren Fragen nach dem Befinden erweitert werden. Auf Kiswahili bieten sich die Fragen nach Familie (Internationalismus: familia), Reise (ebenfalls bekannt: safari), Arbeit (kurzes Wort: kazi) und die Tageszeit Morgen (arabisch, asubuhi, 
für alle, die kein Arabisch können, ein erstes schwieriger zu lernendes Wort) an. Die einzelnen Fragen werden singend im Viererschritt mit entsprechenden Bildkarten nach dem zu Beginn dieses Unterpunktes beschriebenen Muster eingeführt. Die Antwort lautet noch immer: Nzuri tu (sehr gut). Auch können die Bildkarten dann an einzelne TN verteilt werden, die die jeweilige Frage stellen - und der Chor antwortet. Zum Stundenabschluss kann noch einmal das gesamte Lied im Viererschritt gesungen und jetzt auch der Basso Continuo eingeführt werden (Asante sana). Ggf. ist an dieser Stelle bereits mehrstimmiges Singen möglich (1/3 des Kurses Basso Continuo, 1/3 Refrain, 1/3 fragt sich gegenseitig nach dem Namen). Dies hört sich schön an und führt zu einer Art Flow und nach eigener Erfahrung singen selbst diejenigen gerne, die nach eigener Aussage niemals freiwillig singen.

Ein weiteres um Viererschritt früh eingeübtes Lied in meinem Kurs war ein Flexionslied mit dem Thema Hobbys. Dabei wurden unterschiedliche im Präsens regelmäßige Verben, die größtenteils schon aus den TPREinheiten bekannt waren, nach einer Melodie durchkonjugiert (ich singe gern und du schwimmst gern... ). Zum Dichten des Liedes nutzte ich die Melodie des Chants Evening Rise mit seinen verschiedenen Stimmen. Später folgten weitere bereits existente oder umgedichtete Lieder. Alle Lieder wurden im Viererschritt eingeübt und gesungen.

e) Durch Lieder oder andere Tätigkeiten eingeführtes Sprachmaterial kann in Raumläufen eingeübt werden. Hierbei handelt es sich zunächst um einfache Pattern Drills. Nachdem die Frage nach dem Namen und die Antwort per Lied eingeübt sind, können die Lerner*innen durch den Raum gehen und sich gegenseitig nach dem Namen fragen. Anschließend bietet sich eine erste komplexere Interaktion an: Jede/r Lerner*in erhält eine Cue-Karte ${ }^{9}$ mit der Anweisung, sich gegenseitig zu begrüßen und nach dem Namen und dem Befinden zu fragen sowie einer durch ein entsprechende Bild symbolisierte spezielleren Frage aus dem eben erworbenen Pool. Ggf. könnten auch erste aus TPR bekannte Hobbys eingebaut werden. Die Lerner*innen gehen im Raum umher, suchen Partner*innen, stellen und beantworten sich gegenseitig die Fragen, anschließend tauschen sie die Karten und suchen sich eine/n neue/n Partner*in, mit dem sie die Interaktion durchführen.

f) Ab der dritten Woche sind (echte) Rollenspiele in den Unterricht integrierbar, d.h. Kommunikationsaufgaben mit verteilten Rollen, bei denen über Dialogpattern-Drill hinausgehende Spielanreize geschaffen

\footnotetext{
${ }^{9}$ Cue-Karten können Handlungsanweisungen enthalten, Textbausteine oder gar zu sprechende Sätze, aber auch Informationen zur jeweils zu spielenden Figur (Rolle). In unserem Fall war die Handlungsanweisung auf Deutsch formuliert, Anweisungen können aber auch (bei etwas fortgeschritteneren Lernenden) in der Zielsprache formuliert sein oder zur Verfügung stehende Redemittel in der Zielsprache enthalten. Einen schönen Überblick über die Möglichkeiten der Arbeit mit Cue Cards gibt Gedicke 2000.
} 
- Nenne deinen Namen und frage ggf. nach dem Namen deines Partners

- Frage nach dem Befinden und nach Neuigkeiten und beantworte Fragen dazu.

- Dein Fragestichwort:

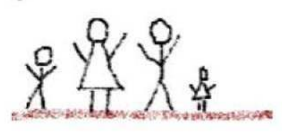

- Dies könnt ihr mehrfach mit anderen Stichwörtern wiederholen, die euch einfallen.

- Tauscht die Karten

- Bedankt euch herzlich beim Partner: Asante sana

- Und geht weiter

- Nenne deinen Namen und frage ggf. nach dem Namen deines Partners

- Frage nach dem Befinden und nach Neuigkeiten und beantworte Fragen dazu.

- Dein Fragestichwort:

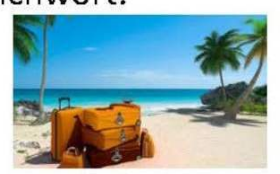

- Dies könnt ihr mehrfach mit jeweils anderen Stichwörtern wiederholen

- Tauscht die Karten

- Bedankt euch herzlich beim Partner: Asante sana

- Und geht weiter

Abbildung 3: Beispiel Cue-Karten zur ersten Partnerinteraktion. Das, wonach speziell zu fragen ist, ist auf dem jeweiligen Bild (entsprechend der vorher eingeführten Bildkarten) zu erkennen.

werden, weil eine Komplikation und ein Überraschungsmoment bereits in der Vorgabe enthalten sind. Beispielhaft demonstriert sei dies anhand der Cue-Card-Konversation „WG-Casting“. Die Lerner*innen in meinem Kurs waren zu diesem Zeitpunkt in der Lage, sich selbst vorstellen zu können, zu sagen, woher sie kommen, ihre Hobbys zu benennen und zu sagen, was sie hassen. Davon ausgehend habe ich Figuren entwickelt: Zwei Personen wohnen in einer WG und suchen jemanden für das dritte Zimmer. Zwei Bewerber stellen sich vor. Jedes Viererset hatte einen eingebauten Konflikt. Dieser bestanden beispielsweise darin, dass aufgrund der Hobbys der/die eine Bewohner*in den/die eine*n Bewerber*in präferieren müsste und der andere den anderen, oder dass die beiden Bewerber*innen viel besser zum/zur eine*n Bewohner*in passen und eigentlich für die ideale WG der/die andere Bewohner*in ausziehen müsste etc. Wichtig für Spiele 
dieser Art ist, dass jeder nur seine eigene Cue-Card kennt, damit sich der Konflikt entwickeln kann. Schon wenn die Spieler*innen diesen allein aufgrund der Interaktion in der Zielsprache irgendwann erkennen, löst dies ein glückliches Aha-Gefühl aus. Die Bearbeitung des Konfliktes auch mit Möglichkeiten des Spiels - löst weitere positive Gefühle aus.

\begin{tabular}{|c|c|}
\hline $\begin{array}{l}\text { A: Du wohnst mit B in der WG. Ihr sucht einen } \\
\text { neuen Mittbewohner. } \\
\text { Dein Name: Emilia } \\
\text { Herkunftsort: Bielefeld, Ujerumani } \\
\text { Unapenda: } \\
\text { Kula } \\
\text { Kusoma } \\
\text { Unachukia: } \\
\text { Kelele } \\
\text { BegrüBt euch, stellt euch vor und tauscht euch } \\
\text { über eure Hobbys aus, und darüber, was ihr hasst. } \\
\text { Wer ist wohl der neue geeignete Mitbewohner? }\end{array}$ & $\begin{array}{l}\text { C: Du suchst ein WG-Zimmer. Bei A und B ist } \\
\text { eines frei. Du stellst dich bei ihnen vor. Du } \\
\text { versuchst, einen guten Eindruck zu machen, } \\
\text { denn du willst das Zimmer. } \\
\text { Dein Name: Asha } \\
\text { Herkunftsort: Moshi, Tanzania } \\
\text { Unapenda: } \\
\text { Kupika } \\
\text { kusoma } \\
\text { Unachukia: } \\
\text { kelele } \\
\text { Begrüßt euch, stellt euch vor und tauscht euch } \\
\text { über eure Hobbys aus, und darüber, was ihr } \\
\text { hasst. } \\
\text { Versuch, das Zimmer zu bekommen. }\end{array}$ \\
\hline $\begin{array}{l}\text { B: Du wohnst mit A in der WG. Ihr sucht einen } \\
\text { neuen Mittbewohner. } \\
\text { Dein Name: Emma } \\
\text { Herkunftsort: Hamburgo, Ujerumani } \\
\text { Unapenda: } \\
\text { Musiki } \\
\text { Kucheza dansi } \\
\text { Unachukia: } \\
\text { kusoma } \\
\text { BegrūBt euch, stellt euch vor und tauscht euch } \\
\text { über eure Hobbys aus, und darüber, was ihr hasst. } \\
\text { Wer ist wohl der neue geeignete Mitbewohner? }\end{array}$ & $\begin{array}{l}\text { D: Du suchst ein WG-Zimmer. Bei A und B ist } \\
\text { eines frei. Du stellst dich bei ihnen vor. Du } \\
\text { versuchst, einen guten Eindruck zu machen, } \\
\text { denn du willst das Zimmer. } \\
\text { Dein Name: Emma } \\
\text { Herkunftsort: Hamburgo, Ujerumani } \\
\text { Unapenda: } \\
\text { Muziki } \\
\text { Kucheza mpira nyumbani } \\
\text { Unachukia: } \\
\text { kusoma } \\
\text { Begrüßt euch, stellt euch vor und tauscht euch } \\
\text { aber eure Hobbys aus, und darüber, was ihr } \\
\text { hasst. Versuch, das Zimmer zu bekommen. }\end{array}$ \\
\hline
\end{tabular}

Abbildung 4: Beispiel Cue-Karten WG-Casting. Die Informationen auf den abgebildeten Rollenkarten sind bis auf Herkunftsorte, Hobbys und Gehasstes noch auf Deutsch. Später lassen sich mehr und mehr Elemente in der Zielsprache integrieren.

In meinem Kurs spielten die Gruppen gleichzeitig, es gab keine Vorbereitungszeit (das hätte dem Überraschungseffekt der Cue Cards 
widersprochen), Vorspielen war nicht geplant. Beim ersten Durchgang beobachtete ich, dass trotz der Spielanlässe bietenden Cue Cards die Gruppen auf zwei Weisen interagierten: Die stärkeren TN fielen sofort wieder in einen Unterrichtsmodus, waren bemüht, alle Sätze korrekt zu formulieren - und ignorierten den Spielanreiz, weil sie ausschließlich mit der Sprachrichtigkeit beschäftigt waren. Die schwächeren Lerner*innen wollten gerne spielen, ihnen fehlten aber die Wörter - Spaß hatten sie auch nicht. Also unterbrach ich das Spiel und stellte eine neue Regel auf: Wenn du etwas sagen möchtest und kannst das nicht auf Kiswahili, so kannst du jede andere Sprache benutzen, so lange niemand anders in der Spielgruppe diese beherrscht. Auch ein plötzlicher Wechsel in eine für alle unbekannte Sprache bietet einen Spielanlass. Weiterhin wies ich darauf hin, dass beim Spielen die Spielfreude das Wichtigste ist, und nicht die Sprachrichtigkeit. Es kommt nicht auf perfekt formulierte Sätze an, sondern darauf zu interagieren - und Spaß zu haben. Beim erneuten Spiel begaben sich alle in die Rollen, die Interaktionen gelangen, die Komplikationen wurden gefunden und spielend ausgehandelt.

Als weitere szenische Spielform mit Sprechanlass wurden Improvisationsaufgaben angeboten und durchgeführt; hierbei beobachtete ich jedoch, dass die eingebrachten Sprechanteile sehr gering waren. Es stellte sich heraus, dass auf diesem Nullanfänger-Niveau alle Lerner*innen zufriedener mit Szenenentwicklungsaufgaben waren, bei denen sie 10 Minuten Vorbereitungszeit hatten. Hierbei war eine größere Varietät an Sprachanteilen bei allen Beteiligten zu beobachten. Tatsächliche Improvisationsaufgaben im Fremdsprachenunterricht, wie beispielsweise bei Walter 2014 angeregt und diskutiert, benötigen vermutlichen einen Sprachstand, der über A1.1-Niveau hinausgeht.

Auch bei den vorbereiteten Szenen in meine Kurs war es aber wichtig, immer wieder daran zu erinnern, dass Spielfreude das Allerwichtigste ist, dass während der Szenen kein Sprachrichtigkeitsfokus vorhanden ist und dass auch das Ausweichen auf andere (allen in der Spielgruppe) fremde Sprachen immer gestattet ist. Mit diesen Vorgaben erhöhten sich letztlich auch die zielsprachlichen Sprechanteile aller sukzessive.

Weitere Spiel- und Sprechanlässe wurden durch von den Lerner*innen selbst geschriebene Texte gewonnen. In meinem Kurs wurde zunächst die poetische Kurzform Elfchen eingeführt; die Hausaufgabe bestand darin, pro Person mindestens ein Elfchen zu verfassen. Die so entstandenen Gedichte wurden in der folgenden Spielsitzung gelesen, vorgelesen, gegenseitig verständlich gemacht, und wiederum als Spielanlass genommen. Von den Lerner*innen verfasste Elfchen waren beispielsweise:

Ausgehend von dieser Schreib- und Spielerfahrung ermunterte ich alle Teilnehmer*innen, Geschichten und Dialoge zu Hause zu schreiben und sie mir zu schicken. Ich verbesserte die Fehler und brachte die Texte als Spielmaterial mit in den Unterricht. Dieses Angebot wurde von einigen 
Tabelle 1: 5 Von den Lerner*innen selbst geschriebene Elfchen

\begin{tabular}{|l|l|}
\hline tisa nane & neun acht \\
saba sita tano & sieben sechs fünf \\
nne tatu mbili moja & vier drei zwei eins \\
ndiyo & ja \\
\hline Haraka & Schnell \\
Wapi choo? & Wo-ist-das Klo? \\
Kwa sauti kubwa & Mit lautem Geräusch \\
ananiita mtoto wangu & ruft-mich mein Kind \\
vizuri & gut \\
\hline Ninakupenda & ich-liebe-dich \\
hewa baridi & Kalte Luft \\
theluji nyeupe laini & weißer weicher Schnee \\
ninatembea na ninajisikia & Ich-gehe und \\
huru & ich-fühle-mich frei \\
masika & Große-Regenzeit \\
nisamehe & jetzt? ich-verstehe-nicht. \\
saa ngapi & sitte \\
sasa? sifahamu. tafadhali & danke langsam danke \\
asante & verzeih-mir \\
\hline & \\
\hline
\end{tabular}


Teilnehmer*innen stärker, von anderen weniger genutzt (was im Sinne des individuellen Lernens ja durchaus akzeptabel ist) und es entstanden einige wirklich gute Geschichten, die wir später im Theaterstück nutzten. Die Arbeit mit den eigenen Texten und Dialogen ${ }^{10}$ förderte das Gefühl der Selbstwirksamkeit der Lerner*innen.

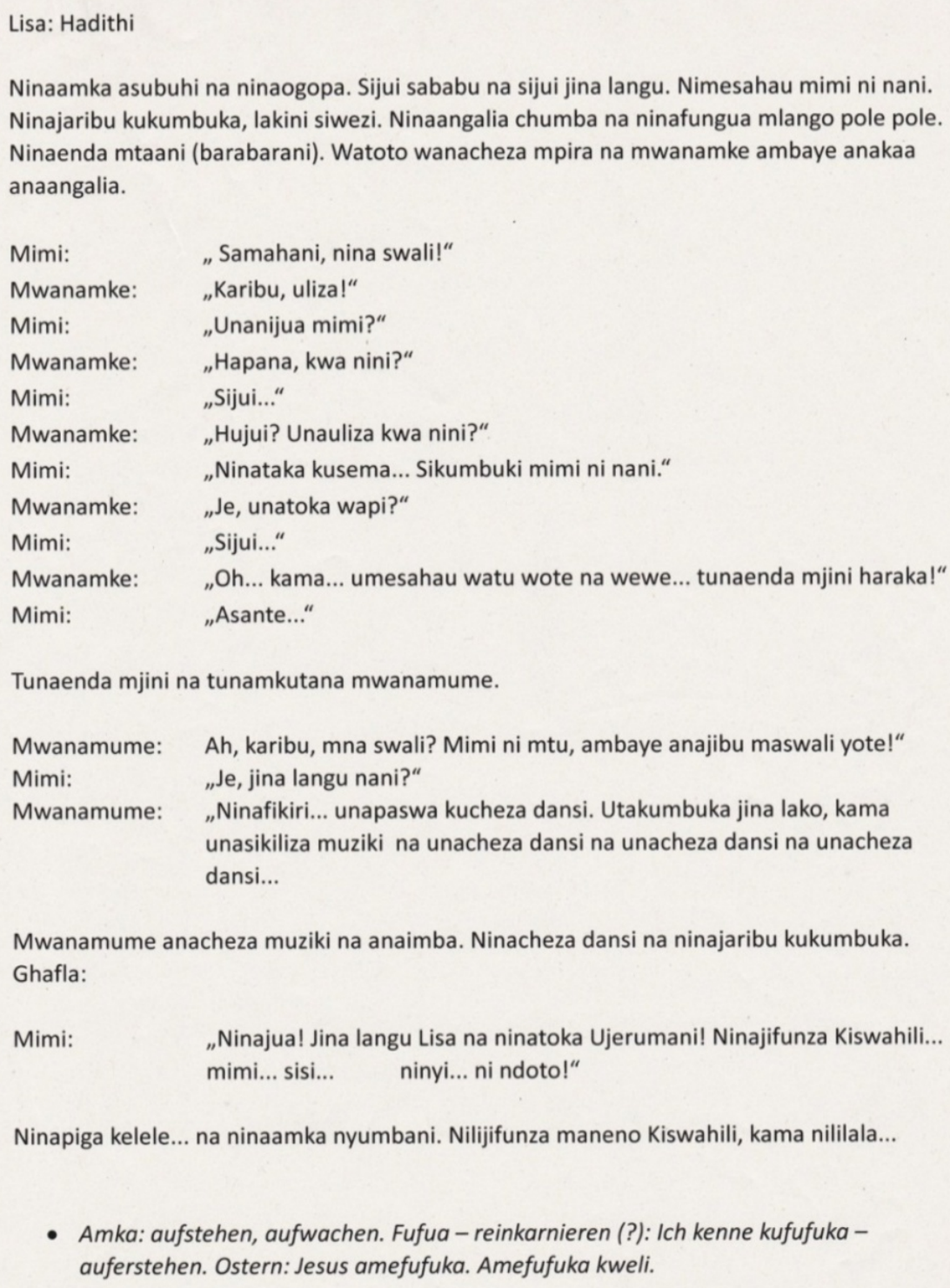

Abbildung 5: Von mir bereits korrigierte Version einer Lernergeschichte zur Frage „Wer bin ich?"11

\footnotetext{
${ }^{10}$ Gute Schreibideen, auch für ein niedriges Sprachniveau in der Zielsprache finden sich in Hippe 2011, insbesondere bei den Einsteigeraufgaben.

${ }^{11}$ Grobe Übersetzung des abgedruckten Beispieltextanfangs: Ich wache morgens auf und ich habe Angst. Ich weiß den Grund nicht und ich weiß meinen Namen nicht. Ich habe vergessen,
} 
Wie sind die oben dargestellten unterschiedlichen Elemente innerhalb einer Unterrichtsstunde nun miteinander zu verzahnen?

\section{Beispielhafter Ablauf einer Spielsitzung (erste Sitzung)}

Das oberste Ziel, ist, dass die Lerner*innen Spaß haben und sich nicht langweilen. Es soll keine Monotonie aufkommen. Deshalb empfiehlt es sich, gerade im Anfängerunterricht, wenn die Aufgaben noch nicht so komplex sein können, fünf bis zehn Minuten-Slots für eine Aktivität anzusetzen. Evtl. werden Aktivitäten zu einem späteren Zeitpunkt in derselben Stunde ggf. leicht modifziert oder erweitert wieder aufgenommen. Die folgende Tabelle zeigt den möglichen Ablauf einer ersten Spielesitzung:

Tabelle 2: Möglicher Ablauf der ersten Sitzung als Beispiel für Abwechslung von Tätigkeiten in Spielsitzungen

\begin{tabular}{|c|c|c|c|}
\hline & (Sozial)form & Inhalt/Ziel & Dauer $^{a}$ \\
\hline 1 & Sprachbad/Demonstration & Aufwärmen: Knuddelpacken & $5-10 \min$ \\
\hline 2 & TPR & mit 4 Verben & $5-10 \min$ \\
\hline 3 & Kreis, Viererschritt, Lied & $\begin{array}{l}\text { Viererschritt einüben und erster Teil des } \\
\text { Liedes }\end{array}$ & $5-10 \mathrm{~min}$ \\
\hline 4 & Raumlauf & sich gegenseitig nach dem Namen fragen & $5 \mathrm{~min}$ \\
\hline 5 & Kreis, Viererschritt, Lied & Refraineinführung & $5-10 \min$ \\
\hline 6 & TPR & $\begin{array}{l}\text { Die ersten } 4 \text { Verben und erste Hobbys (bis } \\
\text { zu weitere } 4 \text { Verben) } b\end{array}$ & $5-10 \min$ \\
\hline 7 & Sprachbad & Wir gehen jetzt auf Bärenjagd & $5-10 \min$ \\
\hline 8 & Kreis, Viererschritt, Lied & $\begin{array}{l}\text { Einführung weitere Fragen nach dem } \\
\text { Befinden mit Hilfe von Bildkarten }\end{array}$ & $5-10 \min$ \\
\hline 9 & $\begin{array}{l}\text { Cue-Card-Dialog/ } \\
\text { Raumlauf }\end{array}$ & $\begin{array}{l}\text { Cue Cards mit Sprechhandlungsaufgaben } \\
\text { zu den bisher eingeführten Redemitteln }\end{array}$ & $5-10 \min$ \\
\hline 10 & Kreis, Viererschritt, Lied & Gesamtes Lied mehrstimmig & $5-10 \mathrm{~min}$ \\
\hline 11 & Einzelarbeit, schriftlich & $\begin{array}{l}\text { Switch auf Deutsch: Schreibt alle Wörter auf, } \\
\text { an die ihr euch erinnert. Auch wenn ihr nur } \\
\text { einen Klang im Kopf habt und glaubt, etwas } \\
\text { könnte ein Wort sein, schreibt es auf }\end{array}$ & $5-10 \mathrm{~min}$ \\
\hline 12 & Plenum & $\begin{array}{l}\text { Abgleich. Die genannten Wörter werden auf } \\
\text { einem Flip-Chart-Papier festgehalten und } \\
\text { erklärt. }\end{array}$ & $5-10 \min$ \\
\hline
\end{tabular}

${ }^{a}$ Die Dauer richtet sich nach der Lust der Lerner*innen. Wenn eine Aktivität anfängt, zu anstrengend oder langweilig zu werden, ist Wechsel angesagt.

${ }^{b}$ Die Schritte 11 und 12 sind nur dann durchführbar, wenn der/die Lehrer*in eine Sprache mit den Lerner*innen teilt.

wer ich bin. Ich versuche, mich zu erinnern, aber ich kann es nicht. Ich gucke das Zimmer an und ich öffne langsam die Tür. Ich gehe auf die Straße. Kinder spielen Ball und eine Frau sitzt da und guckt. Ich: Entschuldigung, ich habe eine Frage. Frau: Gerne, frag. Ich: Kennst du mich? Frau: Nein, warum?... 


\section{Clownsworkshop und Clownsprinzipien ${ }^{12}$}

Wie bereits dem letzten Kapitel zu entnehmen ist, ist die Förderung von Fehlerfreundlichkeit und die Risikofreudigkeit ein zentrales Anliegen des Kurskonzepts. Deshalb wurde der wöchentliche Kursablauf durch den zu Beginn erwähnten Clownsworkshop ergänzt. Nachdem im Kurs eine grundsätzlich positive Gruppendynamik und ein Gruppengefühl etabliert worden waren, bot ich dem Kurs an, einen eintägigen Wochenend-Clownsworkshop durchzuführen, um die Clownsprinzipien zu etablieren. ${ }^{13}$ Das Angebot wurde mit Begeisterung aufgenommen.

Die Clownsnase gilt als die „kleinste Maske der Welt“. Man ist also quasi versteckt, eine andere Person und kann sich dementsprechend mehr Freiheiten erlauben. In den folgenden Spiegelstrichen wird immer jeweils zunächst ein Clownsprinzip vorgestellt und anschließend auf den Fremdsprachenunterricht angewendet:

- Freude am Entdecken, (kindliche) Neugierde. Dies heißt, übertragen auf fremdsprachliches Lernen: Sprache lernen ist Sprache entdecken, mehr wissen wollen und wissen, was man wissen will und das auch einfordern. Es geht also letztlich um induktives und selbstgesteuertes Lernen und Arbeiten.

- Alles ausprobieren, ungewöhnliche Möglichkeiten finden, mit den Dingen spielerisch umgehen. Auch beim Sprachenlernen geht es darum, mit dem, was man hat, zu spielen, es in alle Richtungen zu drehen und zu wenden- und es manchmal auch zu verbiegen. Lerner*innen suchen Anwendungs- und Spiel- bzw. Verfremdungskontexte. Dies löst erstens positive Emotionen aus, zweitens dient es als Mnemotechnik und drittens fördert es die Interaktionsfähigkeit in der Zielsprache: Sprachlich miteinander handeln lernt man dadurch, dass man sprachlich miteinander handelt.

- Zeigen, was IST, kein Vor-Spielen. Auch im Sprach-Spiel ist es wichtig, dies einfach zu machen und zu zeigen, was IST. Überlegt der/die Spielersprecher*in NICHT vor dem Sprachhandeln, was die anderen denken könnten, so kann er freier/weniger gehemmt agieren. Diese Herangehensweise ermöglicht, Ängste und Unwohlsein offen zu zeigen, sie befreit und reduziert die Sprechangst.

- Die Dinge, die SIND, groß machen und sie mit den anderen Spieler*innen (und mit den Zuschauer*innen) teilen. Alles, was man lernen WILL, hat

\footnotetext{
${ }^{12}$ Clownsprinzipien und insbesondere auch die Aktivitäten des Clownsworkshops stammen von Canip Gündogdu, Clown und Ausbilder der LAG Spiel und Theater NRW in Bielefeld zur Förderung von positiven Emotionen in Lernprozessen

${ }^{13}$ Praxishinweis: Um einen Clownsworkshop anzubieten, sollte man unbedingt selbst zumindest an einem Wochenendworkshop teilgenommen haben; man muss die Prinzipien selbst erfahren haben, sonst sind sie nicht vermittelbar.
} 
immer zwei Seiten: Spaß, und damit auch schon verbunden Sinnlichkeit und Lust, aber auch Unsicherheit, Bedrohung, das Verlassen fester Bahnen, Ungewissheit, worauf etwas hinausläuft. Es ist wichtig, all dies und auch die Emotionen, die dadurch ausgelöst werden, zuzulassen und mit den anderen zu teilen. Lernen bedeutet Unsicherheit und positive Herausforderung. Es benötigt einen geschützten Raum, in dem alles möglich ist - Lernen ist immer auch sozial.

- Scheitern, groß Scheitern, Spaß am Scheitern. Mit jedem Experiment verbunden ist immer wieder auch die Möglichkeit des Scheiterns. Wenn uns das im Sprachunterricht nicht mehr peinlich ist, sondern wir das Scheitern als Teil des Spiels begreifen, ist schon viel gewonnen. Auch dieses Prinzip trägt also zur Reduktion von Sprech- und Versagensangst bei.

- Wir haben uns alle lieb. Diese Formulierung ist absichtlich so gewählt: Der Clown weiß, dass er immer, auch wenn er scheitert oder sich streitet, akzeptiert und geliebt wird. Er kann gar nichts wirklich falsch machen. Es geht also um die gegenseitige Gewissheit, dass der andere uns mag und akzeptiert, so wie wir sind, unabhängig davon, welche Fehler wir machen oder welche Konflikte wir austragen. Diese Gewissheit bildet die Basis der gesamten Clownsarbeit. Übertragen auf Sprachunterricht heißt das: Damit all das möglich ist, brauchen wir ein vertrauensvolles soziales Klima in der Lerngruppe.

DerWorkshop selbstwurde aufDeutsch gehalten; dieKiswahili-Sprachkenntnisse der Kursteilnehmer*innen hätten noch nicht ausgereicht. Folgender Ablauf ist für einen solchen Workshop möglich:

- Aufwärmen und Kurzinformation zur Clownsphilosophie

- Bekanntmachung mit der Nase: Rituelles Aufsetzen der Nase, sich selbst und später andere entdecken ${ }^{14}$. Oft verbindet man dieses erste Entdecken mit einem Kurzauftritt vor dem Publikum: Hereinkommen, Publikum ansehen, etwas stehen bleiben, gucken, was passiert. Oft entstehen hier schon bewegende Momente, weil sich in einer solchen Interaktionssituation in der Beziehung zwischen Clown und Publikum Veränderungen ergeben, wenn die Clownsprinzipien beachtet werden. Dadurch, dass der Clown eben nicht vorher plant, wie er auftreten möchte und nur das zeigt, was IST, und dies größer macht, entsteht eine Situationsdynamik zwischen Clown und Publikum. Der Clown tritt durch das Nicht-Spielen ohne den üblichen Selbstschutz auf und wird offen und verletzlich, lässt Neues zu.

\footnotetext{
${ }^{14}$ Der/die Anleitende sollte nicht erschrecken: Dieses allererste Aufsetzen der Nase ist für die Spieler*innen immer irritierend.
} 
- Gegenstände entdecken (mit Nase): Der Raum ist mit gewöhnlichen und ungewöhnlichen Gegenständen ausgelegt. Die Clowns entdecken diese Gegenstände (langsam, behutsam und freudig) - einzelne oder mehrere hintereinander, sie wundern sich und probieren aus, was man mit diesen Gegenständen alles machen kann. Es finden sich ungewöhnlichste Verwendungsweisen, die abschließend in einer kleinen Vorführung gezeigt werden

- Clownstaufe: Alle stehen im Kreis, der Clown, der getauft wird, in der Mitte. Alle rufen sämtliche Namen, die ihnen einfallen, dieser Person zu. Wenn ein Name fällt, der ihr gefällt, sagt diese „Ja!“ und hat damit ihren Clowsnamen. Clownsnamen können im Laufe des Lebens immer dann geändert werden, wenn man das möchte.

- Spiele, bei denen jemand scheitern muss, z. B. Chef - Vize $e^{15}$, ein Zahlenreaktionsspiel, bei dem derjenige, der einen Fehler macht, in der Hierarchie herabgestuft wird und sich langsam wieder hochkämpfen kann. Es geht dabei darum, das Scheitern groß zu machen und genießen zu lernen.

- Tanzen mit und gegen Musik: Musik wird angestellt und der/die Leiter*in sagt an, wie getanzt werden soll (frei, einander imitierend, besonders klein, besonders groß, besonders schön, besonders hässlich etc. - und schließlich NEBEN dem Takt). Die Spieler*innen merken, wie schwer es ist, neben dem Takt zu tanzen. Auch hier können Vorführreihen eingebaut werden. Auch diese Aktivität hat etwas mit der Lust am Scheitern zu tun. Sie führt automatisch zum Freier-Werden: Nach dieser Aktivität tanzt niemand mehr gehemmt.

- Szenenentwicklung unter Berücksichtigung der Clownsprinzipien, Vorführung und Reflexion der Szene: Eine kleine Spielaufgabe wird gegeben. Mit 10-15 Minuten Vorbereitungszeit entwickeln Dreier- oder Vierergruppen eine Szene zu einem vorgegebenen Thema und in einem vorgegebenen Genre, beispielsweise zum Thema „Aus Langeweile wird ein Event“. Genrevorgabe könnte sein: Zirkusnummer oder Musical. Die Szenen werden vorgeführt und von den Zuschauer*innen positiv befeedbackt (Lieblingsmomente etc.).

- Tagesauswertung

In unserem konkreten Fall waren die Teilnehmer*innen vom Tag durchweg begeistert. Die wenigen Personen, die an diesem Tag gefehlt hatten, wurden in der nächsten Spielesitzung eingeführt und getauft und das Clownselement wurde in den späteren Spielesitzungen von ihnen immer wieder aufgegriffen. In den Seminarraumsitzungen hingegen wurde die Clownsnase nicht aufgesetzt,

\footnotetext{
${ }^{15}$ Anleitung siehe http://www.gruppenspiele-hits.de/kreisspiele/ribbel-dibbel.html (28.11.17)
} 
obwohl es eines meiner Ziele gewesen war, bei Unwohlsein/Überforderung etc. die Freiheit $\mathrm{zu}$ geben, in die Clownsrolle zu schlüpfen, um diesem Unwohlsein Ausdruck verleihen und es mit den anderen teilen zu können. Bei der Kursauswertung wurde gesagt, dies sei eben nicht nötig gewesen.

\section{Entwicklung des Kishwahili-Kurses im Laufe des Semesters}

Entsprechend dem Kurskonzept - zu Hause wenig Verpflichtendes, viel Selbstgewähltes - entwickelte sich (wie in jedem Sprachkurs) nach einigen Wochen eine relativ große Leistungsheterogenität. Dies war im Verlauf der Spielesitzungen überhaupt kein Problem - jeder agierte immer entsprechend dem eigenen Sprachstand und entwickelte sich von dort aus weiter. Auch in den Unterrichtsraumsitzungen konnte ich durch innere Differenzierung, Zusatzangebote für Schnellere und Ähnliches gut damit umgehen.

Tatsächlich war ich über die Lernfortschritte einiger Lerner*innen überrascht. Bereits nach wenigen Wochen verstanden diese das meinerseits frei (und nicht besonders langsam) Gesprochene und konnten den schwächeren Lernenden Hilfestellung geben. Auch die selbstgeschriebenen Texte versetzten mich immer wieder in Erstaunen, sie zeigten, wie schnell Sprache gelernt und aktiv in einer neuen Sprache gehandelt werden kann. Zudem blieb die Stimmung durchgängig gelöst und interessiert.

\subsection{Die Krise - und ihre Reflexion}

Das gesamte Semester war also geprägt von Neugier und Spaß auf allen Seiten und - je nach Engagement und auch zu Hause investierter Zeit - unterschiedlich großen Fortschritten. Ich hatte mir vorbehalten, zum letzten Drittel des Kurses zu entscheiden, ob wir als (vom Modul her vorgesehene) Abschlussprüfung eine kleine Aufführung vorbereiten oder eine andere Prüfungsform wählen würden. Da der Kurs und insbesondere dessen Gruppendynamik sich sehr positiv entwickelt hatte, entschied ich mich für die Prüfungsform Probenwochenende und Aufführung nach Abschluss der Vorlesungszeit. Die Reaktion im Kurs war geteilt. Einige hatten Lust zu einer Aufführung, waren aber nicht in Bielefeld, andere trauten sich eine Aufführung nicht $\mathrm{zu}$ - und die Hälfte des Kurses war begeistert. So entschied ich mich, mit dieser Hälfte eine Aufführung einzustudieren und für die anderen eine Klausur anzubieten. An dem der Besprechung folgenden Tag, einem Donnerstag, begannen wir die Stunde, wie üblich, mit der Frage. „Was ist euch von gestern im Kopf geblieben, was wollt ihr wissen?" - und ein allererstes Mal wollte niemand irgendetwas Inhaltliches wissen. Die Stimmung war gedrückt, die Stunde verlief zäh - und ich war sehr beeindruckt davon und bedrückt darüber. Das Problem beschäftigte mich einige Tage und die Überlegungen führen zu folgenden Ergebnissen:

- Klausuren töten jedes potentiell tatsächlich vorhandene Interesse ab. 
Bei zentralen Prüfungen ist es nicht möglich, intrinsische Motivation zu behalten. Ausschließlich die Frage „(Wie) bestehe ich?" ist noch relevant. Die Lähmung im Kurs wurde verursacht durch den Gedankengang: Wenn wir etwas fragen, dann müssen wir das wissen und es ist klausurrelevant - also wollen wir lieber nichts wissen. Die Emotion war zum ersten Mal negativ. Interessant war auch, dass diese Emotion sich auf alle Kursteilnehmer*innen übertrug, auch auf die, die gar keine Klausur schreiben würden, sondern spielen.

- Es würde für mich nicht leicht sein, eine Klausur zu erstellen: Ich hatte den gesamten Kurs über betont, dass jede/r lernen möge, was und wie er/sie will und dass ich erwünschtes Material/erwünschte Aktivitäten bereitstellen würde. Die Gruppe war so sehr heterogen geworden und umfasste die gesamte Spanne von TN, die sich fließend ausdrücken konnten und ganze Texte für unsere Szenen geschrieben hatten, bis hin zu TN, die sich noch immer vorzugsweise in Einzelwörtern ausdrückten, einen eher geringen Wortschatz und nur wenige sprachliche Strukturen zur Verfügung hatten. Entgegen meiner ursprünglichen Absicht war ich gezwungen, nun doch noch eine Art Kanon aufzustellen, eine Liste dessen, was geprüft werden würde. Die Prüfungsform Klausur widerspricht also auch in dieser Hinsicht meinem Unterrichtsprinzip der Autonomie- und Interessenförderung jedes Einzelnen.

Die Probleme und Dilemmata wurden in der darauffolgenden Woche (auf Deutsch) im Kurs reflektiert und so einigermaßen wieder eingefangen. Eine solche transparente Handhabung, von Krisen, die allerdings auf A1Sprachniveau nur mit gemeinsamer Lingua Franca vorhanden ist, halte ich für sehr wichtig. In diesem Fall zeigte sie die unvermeidbare „Dialektik von Autononomie und Heteronomie" (Schmenk 2008: 284): Das institutionell vorgegebene Messen von Leistungen beeinflusst einerseits seitens des Lehrenden die Möglichkeiten, einen Kurs zu gestalten. Andererseits beeinflusst es aber auch die Lernenden hinsichtlich der Motivation und schränkt ihre Möglichkeiten, unabhängig und selbstbestimmt $\mathrm{zu}$ arbeiten ein. Handlungsmöglichkeiten und -beschränkungen sowie sich daraus ergebende gruppendynamische Entwicklungen sollten jeweils mit den Teilnehmer*innen reflekiert werden, um sich der strukturellen Zwänge und der eigenen Handlungsmöglichkeiten bewusst zu werden.

Was in unserem Kurs blieb, war eine Zweiteilung des Kurses in diejenigen, die wieder und weiter engagiert und selbstinteressiert teilnahmen und sich auf Stück und Aufführung freuten und diejenigen, denen etwas mulmig vor der Klausur war, und die auch nicht mehr ganz so viel Spaß an der Sache „Sprache-lernen“ haben konnten. Die Klausur selber wurde geschrieben, von allen bestanden und war in sich unspektakulär, auch einige der Aufführenden schrieben sie - nur so zu Spaß - mit. Evtl. würde ich in einem weiteren Kurs das Format Portfolio als zur Aufführung alternatives, lernprozessbegleitendes Prüfungselement einsetzen. Dieses könnte auch genutzt werden, 
um reflexive Elemente hinsichtlich der Selbsteinschätzung der Entwicklung der eigenen Selbständigkeit, der Effektivität ausprobierter Lernstrategien, der Risikobereitsschaft, etc. zu integrieren. Trotzdem ist der positive Effekt einer Aufführung, wie wir als Theaterpädagog*innen wissen, unschätzbar und bietet den idealen Abschluss eines solchen Kurses.

\subsection{Das Theaterstück und die Aufführung}

Die Aufführung des Stücks, die alternative und von mir bevorzugte Prüfungsform, wurde eine Woche nach Semesterende angesetzt und wurde an drei Probentagen in der ersten Woche der vorlesungsfreien Zeit eingeübt. Es sollte (fast) ausschließlich aus im Kurs bereits genutzten Elementen bestehen. Das hieß, Lieder und weitere chorische Elemente sollten ebenso wie das im Kurs erstellte Text- und Szenenmaterial Bestandteil der Aufführung sein. Weiterhin war mir wichtig, dass jeder Spieler einen Solosprechpart hatte. Schnell stand die Idee fest, dass es ein Clownsstück werden sollte, da die Clownsprinzipien alle TN durchweg begeistert hatten und immer wieder auch Clown-Spielaufgaben eingefordert worden waren. Das Stück wurde in den letzten Wochen des Semesters von der Performance-Künstlerin Zaida Horstmann in Zusammenarbeit mit mir entwickelt.

Die Geschichte war simpel: Ein neugieriger Clown dringt in ein Militärlager ein, wird diskriminiert und weigert sich, den Drill mitzumachen. Es gibt eine Krise, und schließlich werden die Soldaten nacheinander befreit und alle werden $\mathrm{zu}$ (glücklichen, erforschenden, ausprobierenden, freundlichen...) Clowns. Die Sprache wurde dabei nicht nur im realistischen Sinn benutzt, sondern oft absurd: So wurde mit einem aufzusagenden Satz aus einem Kochrezept der Clown diskriminiert, der Chor-Marsch des Militärs bestand aus „Wir gehen jetzt auf Bärenjagd“ etc. Die Solosprechparts wurden mit von den TN jeweils selbstgeschriebenen (und von mir korrigierten) Geschichten bestückt. Die meisten Geschichten wurden erzählt, nachdem die Soldaten „befreit“ worden und wieder zu Individuen geworden waren. Eine der Teilnehmergeschichten wurde handlungstragend ins Stück eingebaut: die weiter oben abgedruckte Geschichte über eine Person, die ihren Namen vergessen hatte.

Das Stück erforderte zwei herausragende Rollen, den Militärchef und den Clown. Als Militärchef wurde eine starke Spielerin gewählt, alle anderen schlüpften nacheinander in die Rolle des Clowns; die Rolle des Protagonisten wurde durch wiederholte rituelle Nasenübergabe im Laufe des Spiels immer weitergegeben, so dass alle Spieler*innen die Gelegenheit hatten, aus der Gruppe herauszutreten.

Ein Problem, mit dem wir uns auseinandersetzen mussten, war die Frage, wie ein Kiswahili-Stück für das Publikum, das zum größeren Teil kein Kiswahili verstand, interessant gestaltet werden konnte. Dabei waren nicht die Elemente, die einen großen Anteil an Körperarbeit und chorischem Sprechen oder Singen enthielten, ein Problem, sondern die Sologeschichten. Wir entschieden uns, dass diese im Stück einem Hörer erzählt werden sollten, der dann immer 
auf Deutsch zentrale Elemente des Erzählten spiegelte. Die Gruppe übte das Stück sehr freudig und diszipliniert ein; anschließend fand die Aufführung vor 50-60 Personen statt. Zum Happy End brachten die Spieler*innen dem gesamten Publikum unser Clownslied bei ${ }^{16}$ und verteilten Clownsnasen, so dass Spieler*innen und Zuschauer*innen glücklich und gelöst den Raum verließen. ${ }^{17}$

\section{Resümee}

Der Kurs hat gezeigt, dass Lernen außerhalb des üblichen (hoch-)schulischen Klassenraumrahmens möglich und möglicherweise sogar effektiver ist als innerhalb dieses Rahmens. Elemente (kindlicher) Neugier können aufgegriffen oder wieder geweckt werden. Nicht zu vergessen ist allerdings, dass wir für unsere Spiel-Arbeit einen Raum zur Verfügung hatten, der bereits suggerierte, dass hier kein normaler Unterricht stattfindet, was das Umschalten in den Spielerahmen sicher erleichtert hat. Heterogenität, in Schule trotz entgegengesetzter Lippenbekenntnisse in den Köpfen der Lehrer*innen noch immer ein Problem, erweist sich in einem Spielrahmen tatsächlich als Ressource - und alle sind überrascht, wie unproblematisch sie tatsächlich ist.

Die Kursauswertung seitens der Lerner*innen fiel durchweg positiv aus. Niemand war der Ansicht, weniger gelernt zu haben als in einem normalen Sprachkurs, die meisten schätzten ihren Lernzuwachs als dauerhafter und größer ein. Alle berichteten, den Kurs genossen zu haben. Allerdings gaben einige Teilnehmer*innen an, aufgrund vieler anderweitiger universitärer Verpflichtungen zu Hause nicht so viel gelernt und geübt zu haben, wie sie es gerne getan hätten. Auch diese Gegebenheit illustriert sehr schön, dass der existente gesellschaftliche Rahmen Handlungsmöglichkeiten immer mit beeinflusst (s.o.: „Dialektik von Autonomie und Heteronomie“, Schmenk).

Die Krise hat ebenfalls gezeigt, wie strukturelle Zwänge Eigeninitiativen hemmen können. Man lernt besser das, was man lernen WILL - wenn aber das gesamte System auf Druck und Kontrolle aufgebaut ist, fällt u. U. das Engagement für die Veranstaltung in der am wenigsten Druck aufgebaut wird, ${ }^{18}$ hintenüber. Die Schlussfolgerung daraus ist $\mathrm{m}$. E. nicht, dass man sich als Lehrer*in ans System anpassen sollte, sondern dass man immer wieder betonen sollte, dass das normorientierte, homogenisierende, System, in dem Wissen über Klausuren abgetestet wird, eben nicht das lerngünstigste ist und dass Elemente alternativen Lernens in Unterricht integriert werden sollten, um längerfristig das System wieder etwas menschen- und lernfreundlicher und emanzipatorischer zu gestalten. Unterricht ist demokratische Bildung! - oder eben nicht.

In meinen Augen ist ein Pädagoge dann ein guter Pädagoge, wenn er

\footnotetext{
${ }^{16}$ Es handelte sich dabei um unser Verb-Deklinationslied im Präsens.

${ }^{17}$ Links zur Videodokumentation der drei Szenen befinden sich im Anhang.

${ }^{18}$ Siehe dazu auch Schmenk 2008: 280ff.
} 
begriffen hat, dass sein Handeln immer politisches Handeln ist und er Schritt für Schritt bewusster seine Aufgaben in engagierter Weise wahrnimmt. (Nix 2013: 48)

\section{Bibliographie}

Bohle, Kirsten (2011/2017): Theatermethoden für den DaF/DaZ-Bereich: Konzept zur Nutzung theaterpädagogischer Methoden im Fach Deutsch als Fremd- und Zweitsprache. Online-Publikation der Universität Bielefeld: https://pub.uni-bielefeld.de/publication/2909426, eingesehen am 23.05.2017

Gedicke, Monika (2000): Rollenspiele im Fremdsprachenunterricht - eine Möglichkeit zur Förderung realitätsbezogener Kommunikation? In: fsu 44/53 82000, 22-28

Haftner, Magdalena \& Kuhfuß, Anne-Marie (2014): Ich hab gar nicht gemerkt, dass ich Deutsch spreche - Wie die theatrale Erfahrung des Improvisationstheaters freies und authentisches Sprechen erlebbar macht und die kommunikative Kompetenz fördert. In: Bernstein, Nils; Lerchner, Charlotte (Hg.) (2014): Ästhetisches Lernen im DaF-/DaZ-Unterricht. Literatur - Theater - Bildende Kunst -Musik - Film. Materialien Deutsch als Fremdsprache 93, Göttingen: Universitätsverlag Göttingen, 217-231

Hippe, Lorenz (2011): Und was kommt jetzt? Szenisches Schreiben in der theaterpädagogischen Praxis. Weinheim: Deutscher Theaterverlag Holec, Henri (1958): Autonomy and foreign language learning. Oxford: Pergamon Press

Horstmann, Susanne (2014): Abschlussbericht Kiswahili mit Theatertechniken Unveröffentlicht, (Auf Wunsch per E-Mail)

Johnstone, Keith (1993): Improvisation und Theater. Berlin: Alexander Küppers, Almuth; Walter, Maik (2012): Theatermethoden auf dem Prüfstand der Forschung: Einführung in die Themenausgabe. In: Scenario 1/2012 http://publish.ucc.ie/scenario/2012/01/kuepperswalter/01/de, eingesehen am 07.12.2017

Nix, Christof (2012): Theaterpädagogik oder müssen wir nicht erst einmal die herrschende Pädagogik in Frage stellen. In: ders.; Sachser, Dietmar; Streisand, Marianne (Hg.): Lektionen Theaterpädagogik 5. Berlin: Theater der Zeit, 45-52 Sambanis, Michaela (2013): Fremdsprachenunterricht und Neurowissenschaften. Tübingen: Narr

Schmenk, Barbara (2008): Lernerautonomie. Karriere und Sloganisierung eines Begriffs. Tübingen: Narr

Teymoortash, Neda (2010): Effizienz in der Wortschatzvermittlung. Marburg: Tectum Verlag Walter, Maik (2014): Mit Worten Räume bauen: Improvisationstheater und szenische Wortschatzvermittlung. In: Bernstein, Nils; Lerchner, Charlotte 
(Hrsg.): Ästhetisches Lernen im DaF-/DaZ-Unterricht. Literatur - Theater Bildende Kunst-Musik - Film. Materialien Deutsch als Fremdsprache 93,

Göttingen: Universitätsverlag Göttingen, 233-247 


\title{
A Anhang
}

\section{A.1 Links zur Illustration des Kurses}

\author{
http://www.uni-bielefeld.de/lili/studium/faecher/daf/personen/horstmann_ \\ susanne/kiswahili_theatertechniken.html \\ https://www.youtube.com/watch?v=V4awce0IH8c \\ Ausschnitte aus dem Theaterstück: \\ Diskriminierungsszene: https://vimeo.com/235894189, Password: Kiswahi- \\ li \\ Redeanteil: https://vimeo.com/235888439, Password: Kiswahili \\ Abschlusslied: https://vimeo.com/235882291, Password: Kiswahili
}

\section{A.2 Tipps für Lehrer*innen}

a) Zentral für das Gelingen eines Spiel-Sprachkurses ist die Etablierung eines Spiel-Rahmens für Spiel-Sitzungen bzw. Spiel-Sequenzen. Dazu gehören verschiedene Bestandteile

- der Raum. Ideal ist ein Raum außerhalb des üblichen Unterrichtsraums ohne Tische und Stühle. Der übliche Unterrichtsraum sollte umgestaltet werden (Tische und Stühle an den Rand) ${ }^{19}$.

- das Aufwärmen, das am leichtesten durch körperlich anstrengende Spiele herzustellen ist. Später können bei Bedarf auch Techniken wie Traumreise, Massage etc. genutzt werden.

- die Rolle des Kursleiters/der Kursleiterin. Dieser sollte versuchen, die übliche Lehrerrolle so weit wie möglich abzulegen, d.h. mitspielen, selbst auch Risiken eingehen, und insbesondere sich als Spieler lächerlich machen, wenn dies von den TN erwartet wird ${ }^{20}$. Der Teacher in Role kann als Vorbild dazu beitragen, dass Körper und Körperlichkeit exzessiver genutzt werden.

- die Betonung, dass der Sinn des Spiels das Spiel ist. In Sprachkursen verfallen Lerner*innen immer wieder schnell in den Unterrichtsmodus. Der ist aber für die Spielesitzungen kontraproduktiv. Der/die Lehrer*in muss also immer wieder daran erinnern, dass nicht die sprachliche Korrektheit wichtig ist, sondern das Spiel und der Spaß daran. Arbeit an der sprachlichen Form findet phasenweise in der Unterrichtssitzung statt.

b) Innerhalb der Spielsitzungen wird vom Lehrenden (fast) durchgängig die Zielsprache benutzt. Arbeitsanweisungen werden wiederholt, demonstriert, der gesamte Körper wird benutzt. Ggf. helfen die TN sich gegenseitig beim Verständnis der Aufgaben.

\footnotetext{
19 Siehe Bohle 2011/2017

${ }^{20}$ Siehe Johnstone 1993.
} 
c) Folgender Umgang mit Problemen hat sich als sinnvoll erwiesen: Wenn eine Aufgabe oder Übung nicht klappt, nicht mit Freude bearbeitet wird, ist es sinnvoll, diese zu unterbrechen und neue Regeln aufzustellen, Regeln, die mehr Freiheit lassen (siehe Probleme erstes Rollenspiel), Ausweichmöglichkeiten aufzuzeigen - und wiederum in Erinnerung zu rufen, dass Spaß das allerwichtigste ist und Scheitern zum Spiel dazugehört (siehe Clownsprinzipien). 